\title{
Mortality prediction of serum neutrophil gelatinase-associated lipocalin in patients requiring continuous renal replacement therapy
}

\author{
Yohan Park ${ }^{1, *}$, Tae Hyun Ban ${ }^{2,}$, Hyung Duk Kim ${ }^{1}$, Eun Jeong Ko ${ }^{1}$, Jongmin Lee ${ }^{3}$, Seok Chan Kim³, \\ Cheol Whee Park ${ }^{1}$, Chul Woo Yang ${ }^{1}$, Yong-Soo Kim ${ }^{1}$, and Byung Ha Chung ${ }^{1}$
}

\begin{abstract}
${ }^{1}$ Division of Nephrology, Department of Internal Medicine, Seoul St. Mary's Hospital, College of Medicine, The Catholic University of Korea, Seoul; ${ }^{2}$ Division of Nephrology, Department of Internal Medicine, Eunpyeong St. Mary's Hospital, College of Medicine, The Catholic University of Korea, Seoul; ' ${ }^{3}$ Division of Pulmonary, Allergy and Critical Care Medicine, Department of Internal Medicine, Seoul St. Mary's Hospital, College of Medicine, The Catholic University of Korea, Seoul, Korea
\end{abstract}

Received: December 29, 2019 Revised : February 4, 2020

Accepted: February 5, 2020

\section{Correspondence to}

Byung Ha Chung, M.D.

Division of Nephrology,

Department of Internal

Medicine, Seoul St. Mary's

Hospital, College of Medicine,

The Catholic University of Korea,

222 Banpo-daero, Seocho-gu,

Seoul 06591, Korea

Tel: +82-2-2258-6851

Fax: +82-2-2258-6917

E-mail: chungbh@catholic.ac.kr

https://orcid.org/0000-0003-

0048-5717

*'These authors contributed

equally to this work.
Background/Aims: We investigated whether serum neutrophil gelatinase-associated lipocalin (NGAL) can predict mortality in patients with acute kidney injury (AKI) requiring continuous renal replacement therapy (CRRT).

Methods: This study enrolled 169 patients who underwent serum NGAL testing at CRRT initiation from June 2017 to January 2019. The predictive power of serum NGAL level for 28-day mortality was compared to the Acute Physiology and Chronic Health Evaluation-II (APACHE-II) score and Sequential Organ Failure Assessment (SOFA) score via area under the receiver operating characteristic curve (AuROC) value.

Results: There were 55 survivors and 114 non-survivors at 28 days post-CRRT initiation. Median serum NGAL level was significantly higher in the non-survivor group than in the survivor group $(743.0 \mathrm{ng} / \mathrm{mL}$ vs. $504.0 \mathrm{ng} / \mathrm{mL}, p=0.003)$. The AuROC value of serum NGAL level was 0.640, which was lower than APACHEII score and SOFA score values ( 0.767 and 0.715 , respectively). However, in the low APACHE-II score group (<27.5), AuROC value of serum NGAL was significantly increased (0.698), and it was an independent risk factor for 28 day-mortality (hazard ratio, 2.405; 95\% confidence interval, 1.209 to $4.783 ; p=0.012$ ).

Conclusions: In patients with AKI requiring CRRT, serum NGAL levels may be useful for predicting short-term mortality in those with low APACHE-II scores.

Keywords: Neutrophil gelatinase-associated lipocalin; Critical illness; Acute kidney injury; Renal replacement therapy; Mortality

\section{INTRODUCTION}

Neutrophil gelatinase-associated lipocalin (NGAL) is a member of the lipocalin superfamily and is known as an excellent marker for early diagnosis of acute kidney injury (AKI) because it increases early in the process and acts as an indicator of injury rather than loss of renal function [1-3]. Additionally, there have been recent stud- 
ies that report increased expression of NGAL both in renal and extra-renal tissue in various pathologic conditions [3]. In the sepsis-induced mouse model, the expression of NGAL increased not only in the kidney but also in other organs such as the lung, liver, spleen, and trachea [4]. A human study demonstrated that serum NGAL levels increased by inflammatory cell secretion during infection [5]. Therefore, serum NGAL concentration is not only an excellent marker for predicting AKI but also increases its expression in various organs in many pathologic conditions.

NGAL expression has also been shown to be useful in predicting clinical outcomes in both renal and non-renal organ injury. For example, serum NGAL levels are an early biomarker of delayed graft function and calcineurin inhibitor nephrotoxicity after kidney transplant [6], and Cruz et al. [7] reported that serum NGAL levels were elevated in patients with cardiovascular disease. In addition, serum NGAL levels are an independent marker for predicting 3-year mortality in acute heart failure [8] and have been reported to increase with atherosclerosis [9]. Therefore, it can be used to predict mortality in patients with critical illness by reflecting dysfunction of various organs.

Previous studies have shown that NGAL levels can predict overall mortality in patients with critical illness, however, most of those studies were performed in patients with sepsis or in postoperative status [10-13]. A mortality analysis study focusing on serum NGAL levels in critically ill patients with AKI was a small study, involving only 50 patients [13]. The aim of the study presented here is to investigate whether serum NGAL levels can be used to predict mortality in critically ill patients with AKI requiring continuous renal replacement therapy (CRRT) irrespective of underlying cause.

\section{METHODS}

\section{Study design}

This was a retrospective, observational study to analyze critically ill patients with AKI requiring CRRT who were admitted to Seoul St. Mary's Hospital between June 2017 and January 2019. AKI was diagnosed according to the Kidney Disease: Improving Global Outcomes (KDIGO) guidelines [14]. CRRT was initiated in patients with clin- ical hemodynamic instability with volume overload, electrolyte imbalance, and metabolic acidosis that could not be controlled by intensive medical treatment. The protocol for CRRT initiation in our institution was followed, and it starts with continuous venovenous hemodiafiltration at an effluent rate of $35 \mathrm{~mL} / \mathrm{kg}$ per hour. A total of 545 patients underwent CRRT during the study period, of which 41 patients were excluded because they were already undergoing maintenance hemodialysis. An additional 335 patients were excluded because NGAL measurements were not performed in them at the time of CRRT initiation. The remaining 169 patients were enrolled and analyzed in our study.

This study followed the Declaration of Helsinki and was approved by the Institutional Review Board of Seoul St. Mary's Hospital (KC19RASIo780). Written informed consent by the patients was waived by the board due to a retrospective nature of our study.

\section{Data collection}

Baseline patient characteristics were recorded and included age, height, weight, sex, comorbidities, and main indications for CRRT initiation. Blood tests were performed to measure complete blood cell counts, blood urea nitrogen, creatinine, electrolytes, bilirubin, C-reactive protein, and arterial blood gas analysis. Acute Physiology and Chronic Health Evaluation-II (APACHE-II) scores [15] and Sequential Organ Failure Assessment (SOFA) scores [16] were calculated based on patients' vital signs and blood tests at the time of CRRT initiation. We also recorded whether mechanical ventilation was used and whether inotropic agents were administered at the time of CRRT initiation.

\section{Measurement of serum NGAL levels}

Serum NGAL measurements were performed on the sample just before CRRT initiation using BioPorto Diagnostics' NGAL Test Reagent kit (Bioporto Diagnostics A/S, Hellerup, Denmark). Blood samples were collected in tubes containing ethylenediaminetetraacetic acid, and serum NGAL measurements were taken within 30 minutes of collection per the manufacturer's instruction. In this measurement method, the assay range of serum NGAL is 50 to $3,000 \mathrm{ng} / \mathrm{mL}$, and the inaccuracy is less than $5 \%[17]$. 


\section{Statistical analysis}

All continuous variables were expressed as mean \pm standard deviation or median (interquartile range). The Student $t$ test was used to compare variables showing normal distribution and the Mann-Whitney $U$ test was used for variables showing non-normal distribution. Nominal variables were compared using the chi-square test or Fisher's Exact test as appropriate and expressed as proportions. Survival analysis at 28 days post CRRT initiation was represented by Kaplan-Meier curves, and the comparison between the groups was performed by log-rank test. Serum NGAL levels, APACHE-II scores, and SOFA scores were analyzed as 28-day mortality predictors using receiver operating characteristic (ROC) curves. Cutoff values were determined to represent the highest sensitivity and specificity simultaneously [18]. Spearman's correlation analysis was used to investigate the relationship between serum NGAL levels, APACHEII scores, and SOFA scores. Patients were divided into subgroups according to the cutoff value of the APACHEII score. Serum NGAL levels, APACHE-II scores, and SOFA scores were analyzed for 28-day mortality predictors using ROC curves in the subgroup of patients with low APACHE-II scores. Cox proportional hazard regression analysis was performed to identify risk factors for 28-day mortality in the low APACHE-II score group. A $p$ values less than 0.05 were considered statistically significant, and all statistical analyses were performed using SPSS statistics version 24.o (IBM Co., Armonk, NY, USA) and Microsoft Excel 2016 (Microsoft, Redmond, WA, USA).

\section{RESULTS}

\section{Baseline characteristics}

At 28 days after CRRT initiation, 55 patients belonged to the survivor group and 114 patients had not survived. Table 1 shows the baseline characteristics and clinical features in both groups. The mean age was $59.3 \pm 16.2$ years in the survivor group but was significantly higher in the non-survivor group at $65.3 \pm 15.1$ years $(p=0.019)$. There were also significant differences between the survival group and the non-survival group in median heart rate (100.0 bpm vs. $120.0 \mathrm{bpm}, p<0.001)$, median respiratory rate (21.0 bpm vs. $25 \mathrm{bpm}, p=0.007$ ), use of me- chanical ventilation ( $45.5 \%$ vs. $78.9 \%, p<0.001$ ), administration of inotropic agents ( $58.2 \%$ vs. $81.6 \%, p=0.001$ ), mean arterial pressure $(76.3 \mathrm{mmHg}$ vs. $68.0 \mathrm{mmHg}, p$ $<0.001$ ), and arterial pH (7.29 vs. 7.39, p < 0.001). These factors were all included in calculating the APACHE-II scores and SOFA scores. Except for sepsis, the indication for CRRT initiation did not have a significant impact on survival, nor did any of the comorbidities included in this study.

\section{Predictive value for mortality of serum NGAL levels, APACHE-II scores, and SOFA scores}

Serum NGAL levels were significantly higher in the non-survivor group than in the survivor group (median $743.0 \mathrm{ng} / \mathrm{mL}$ and mean 1,033.3 ng/mL vs. median 504.0 $\mathrm{ng} / \mathrm{mL}$ and mean $709.9 \mathrm{ng} / \mathrm{mL}, \mathrm{p}=0.003)$. The APACHEII score (31.0 vs. 24.0, $p<0.001$ ) and the SOFA score (14.0 vs. 11.0, $p<0.001)$ were also both significantly higher in the non-survivor group (Fig. 1). The serum NGAL levels, APACHE-II scores, and SOFA scores were analyzed using ROC curves as predictors of 28 day-mortality. The area under the ROC curve (AuROC) value of APACHE-II score was the highest at $0.767(p<0.001)$, and when the cutoff value was set to 27.5 , the sensitivity and specificity were $68.4 \%$ and $70.9 \%$, respectively. The AuROC values of the SOFA score $(0.715, p<0.001)$ and serum NGAL level (o.640, $p=0.003$ ) were statistically significant. When the cutoff value of serum NGAL was set to $576.5 \mathrm{ng} / \mathrm{mL}$, the sensitivity and specificity were $60.5 \%$ and $58.2 \%$, respectively (Fig. 2).

\section{Correlation analysis of serum NGAL levels, APACHE- II scores, and SOFA scores and subgroup analysis in the low APACHE-II score group}

In the correlation analysis between serum NGAL levels, APACHE-II scores, and SOFA scores, APACHE-II scores and SOFA scores showed a relatively linear correlation with a Spearman's correlation coefficient $(\rho)$ of 0.647 . However, serum NGAL levels did not show clear correlations with either APACHE-II scores or SOFA scores, of which Spearman's $\rho$ values were 0.235 and 0.196 , respectively (Fig. 3). The patients were then divided into high and low APACHE-II score groups according to the APACHE-II cutoff value (Supplementary Tables 1 and 2). There were 94 patients in the high APACHE-II score group and 75 in the low APACHE-II score group. Serum 
Table 1. Baseline characteristics and clinical features of survivor and non-survivor group

\begin{tabular}{|c|c|c|c|}
\hline Characteristic & Survivor $(\mathrm{n}=55)$ & Non-survivor $(n=114)$ & $p$ value \\
\hline Age, yr & $59 \cdot 3 \pm 16.2$ & $65.3 \pm 15.1$ & 0.019 \\
\hline Body mass index, $\mathrm{kg} / \mathrm{m}^{2}$ & $23.8 \pm 4.1$ & $22.9 \pm 3.6$ & 0.159 \\
\hline Male sex & $31(56.4)$ & $65(57.0)$ & 0.936 \\
\hline Mean arterial pressure, $\mathrm{mmHg}$ & $76.3(69.0-92.0)$ & $68.0(60.0-79.7)$ & $<0.001$ \\
\hline Heart rate, bpm & $100.0(78.0-120.0)$ & $120.0(103.3-132.3)$ & $<0.001$ \\
\hline Respiratory rate, rpm & $21.0(18.0-27.0)$ & $25.0(20.0-29.0)$ & 0.007 \\
\hline Body temperature, ${ }^{\circ} \mathrm{C}$ & $36.9(36.4-37.5)$ & $36.8(36.3-37.8)$ & 0.708 \\
\hline Arterial $\mathrm{pH}$ & $7.39(7.33-7.45)$ & $7.29(7.17-7.38)$ & $<0.001$ \\
\hline Serum sodium, mEq/L & $138.5 \pm 8.4$ & $141.1 \pm 8.7$ & 0.071 \\
\hline Serum potassium, $\mathrm{mEq} / \mathrm{L}$ & $4.0(3.5-4.4)$ & $4.2(3.7-4.9)$ & 0.040 \\
\hline Serum creatinine, mg/dL & $2.87(1.77-3.89)$ & $2.40(1.76-3.82)$ & 0.293 \\
\hline Hematocrit, \% & $27.5 \pm 5.9$ & $27.7 \pm 5.7$ & 0.849 \\
\hline WBC count, $\times 10^{3} / \mu \mathrm{L}$ & $12.2(7.6-21.1)$ & $9.7(2.1-18.8)$ & 0.182 \\
\hline Platelet count, $\times 10^{3} / \mu \mathrm{L}$ & $76.0(43.0-166.0)$ & $59.5(29.8-104.0)$ & 0.088 \\
\hline Total bilirubin, mg/dL & $1.85(0.75-5.49)$ & $1.89(0.90-3.64)$ & 0.846 \\
\hline C-reactive protein, mg/dL & $11.4(2.8-19.0)$ & $11.8(5.0-19.7)$ & 0.641 \\
\hline Mechanical ventilation & $25(45 \cdot 5)$ & $90(78.9)$ & $<0.001$ \\
\hline Inotropics administration & $32(58.2)$ & $93(81.6)$ & 0.001 \\
\hline \multicolumn{4}{|l|}{ Indication for CRRT initiation } \\
\hline Sepsis & $22(40.0)$ & $71(62.3)$ & 0.006 \\
\hline Post-operation & $8(14.5)$ & $6(5 \cdot 3)$ & 0.069 \\
\hline Cardiac & $5(9.1)$ & $12(10.5)$ & 0.771 \\
\hline Pulmonary & $1(1.8)$ & $2(1.8)$ & 1.000 \\
\hline Hepatic & $10(18.2)$ & $10(8.8)$ & 0.076 \\
\hline Renal & $4(7.3)$ & $5(4 \cdot 4)$ & 0.475 \\
\hline Malignancy & $5(9.1)$ & $6(5 \cdot 3)$ & 0.340 \\
\hline Others & o & $2(1.8)$ & 1.000 \\
\hline \multicolumn{4}{|l|}{ Comorbidities } \\
\hline Diabetes mellitus & $18(32.7)$ & $28(24.6)$ & 0.264 \\
\hline Hypertension & $21(38.2)$ & $53(46.5)$ & 0.308 \\
\hline Chronic kidney disease & $9(16.4)$ & $12(10.5)$ & 0.281 \\
\hline Cerebro-cardiovascular disease & $17(30.9)$ & $28(24.6)$ & 0.382 \\
\hline Pulmonary disease & $2(3.6)$ & $9(7.9)$ & 0.507 \\
\hline Liver disease & $16(29.1)$ & $18(15.8)$ & 0.043 \\
\hline Dementia & o & $2(1.8)$ & 1.000 \\
\hline Malignancy & $13(23.6)$ & $29(25.4)$ & 0.234 \\
\hline
\end{tabular}

Values are presented as mean $\pm \mathrm{SD}$, number (\%), or median (interquartile range).

WBC, white blood cell; CRRT, continuous renal replacement therapy.

NGAL levels, APACHE-II scores, and SOFA scores were analyzed using ROC curves as predictors of 28-day mortality in the low APACHE-II score group. The AuROC values for prediction of 28-day mortality of serum NGAL levels, APACHE-II scores, and SOFA scores were 0.698, 0.668 and 0.643 , respectively. Using a serum NGAL lev- 

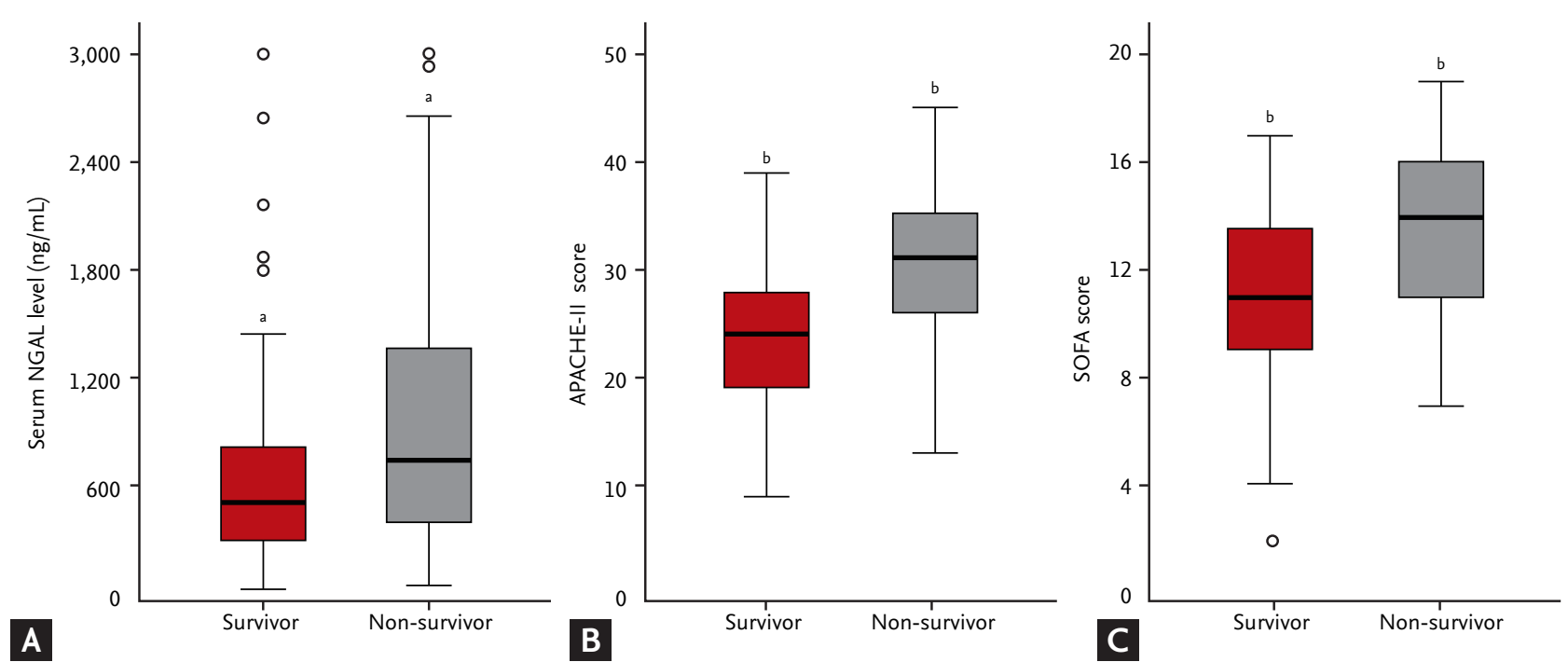

Figure 1. Comparison of median serum (A) neutrophil gelatinase-associated lipocalin (NGAL) level, (B) Acute Physiology and Chronic Health Evaluation-II (APACHE-II) score, and (C) Sequential Organ Failure Assessment (SOFA) score between survivor and non-survivor group. Median serum NGAL level was $504.0 \mathrm{ng} / \mathrm{mL}$ in the survivor group and $743.0 \mathrm{ng} / \mathrm{mL}$ in the non-survivor group $(p=0.003)$. Median APACHE-II score was 24.0 in the survivor group and 31.0 in the non-survivor group ( $p<0.001)$. Median SOFA score was 11.0 in the survivor group and 14.0 in the non-survivor group $(p<0.001) .{ }^{a} p=0.003,{ }^{b} p<0.001$.

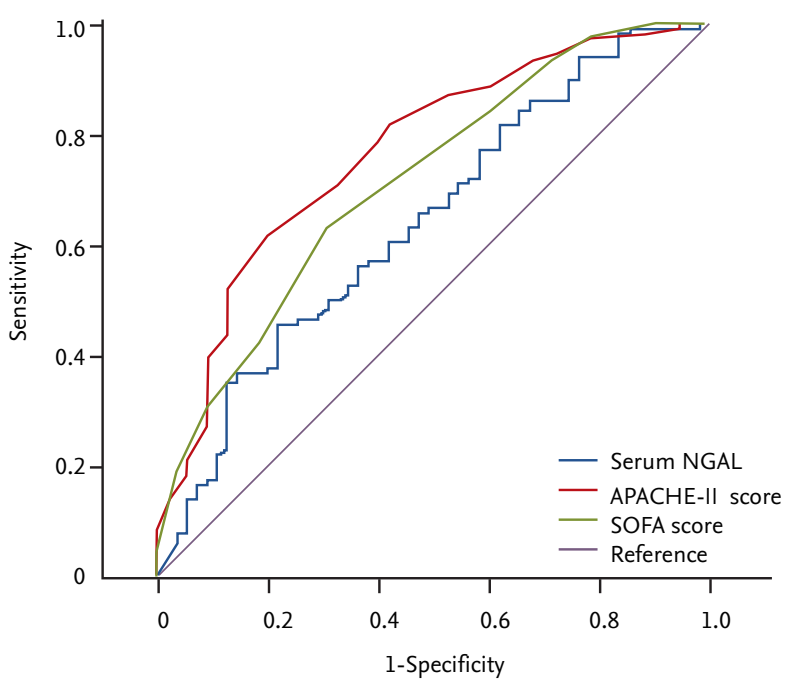

$\begin{array}{rcrrcc} & \text { AuROC value } & p \text { value } & \text { Cutoff } & \text { Sensitivity } & \text { Specificity } \\ \text { Serum NGAL } & 0.640 & 0.003 & 576.5 & 60.5 \% & 58.2 \% \\ \text { APACHE-II score } & 0.767 & <0.001 & 27.5 & 68.4 \% & 70.9 \% \\ \text { SOFA score } & 0.715 & <0.001 & 12.5 & 63.2 \% & 69.1 \%\end{array}$

Figure 2. Predictive value for 28 -day mortality of serum neutrophil gelatinase-associated lipocalin (NGAL) level, Acute Physiology and Chronic Health Evaluation-II (APACHE-II) score, and Sequential Organ Failure Assessment (SOFA) score. AuROC, area under the receiver operating characteristic curve. el cutoff value of $511.5 \mathrm{ng} / \mathrm{mL}$, sensitivity was $63.9 \%$ and specificity was $66.7 \%$. These results indicate that serum NGAL levels showed a rather high predictive power trend compared to other scoring systems, and the predictive power tended to increase in the low APACHE-II score group (Fig. 4).

\section{Combined analysis of NGAL level and APACHE-II score for the prediction of patient survival}

Kaplan-Meier survival analysis was performed all the patients by dividing into four groups according to the cutoff values of APACHE-II score and serum NGAL level. When the APACHE-II score was high, there was no significant difference in mortality rate as serum NGAL levels changed. However, when the APACHE-II score was low, mortality rate was significantly higher in the group with higher serum NGAL levels $(p<0.001$ ) (Fig. 5). Cox proportional hazard regression analysis was performed to identify factors affecting 28-day mortality in patients with low APACHE-II scores. Independent factors affecting 28-day mortality were serum NGAL levels and APACHE-II scores. The hazard ratios of serum NGAL levels and APACHE-II scores were 2.405 and 2.291, respectively (Table 2). 


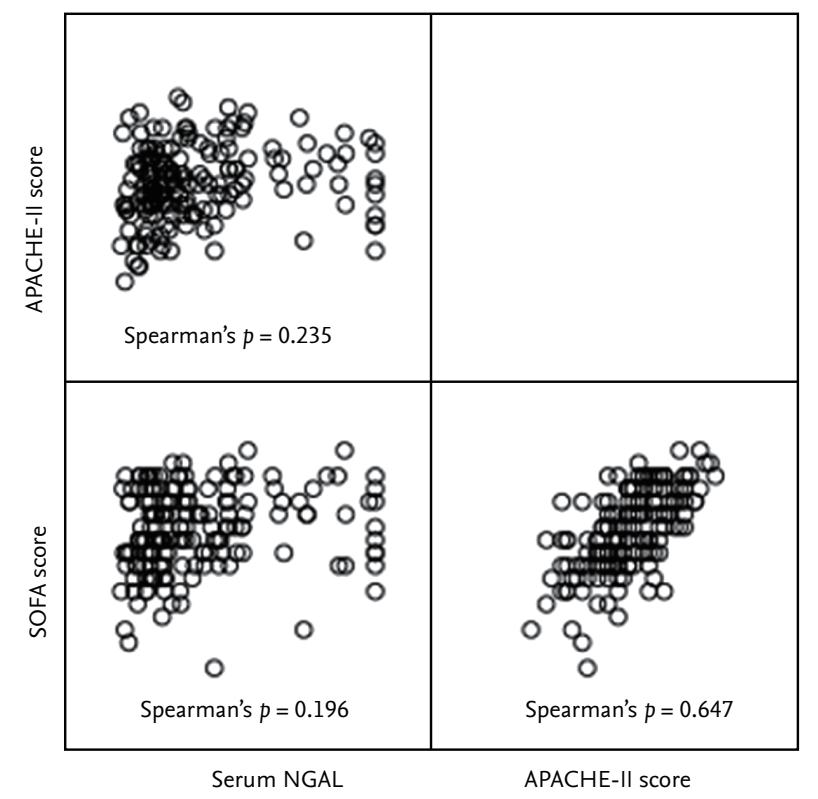

Figure 3. Analysis of correlation between serum neutrophil gelatinase-associated lipocalin (NGAL) level, Acute Physiology and Chronic Health Evaluation-II (APACHE-II) score, and Sequential Organ Failure Assessment (SOFA) score.

\section{DISCUSSION}

In this study, we found that serum NGAL concentration can be a useful predictive marker of mortality in patients with a low APACHE-II score. It was also an independent risk factor for 28-day mortality in the low APACHE-II score group. However, there was no correlation between serum NGAL levels and mortality in the high APACHEII score group.

The 28-day mortality rate of the patients who were enrolled in this study was $67.5 \%$. Considering the possibility of selection bias, baseline characteristics were compared with 335 patients who underwent CRRT but not NGAL measurement. The 28-day mortality rate of the excluded patient group was $65.7 \%$, which was not significantly different from the study group, and there was no significant difference in other parameters between the two groups (Supplementary Table 3). Additionally, the mean APACHE-II and SOFA scores of patients in this study were $28.3 \pm 7.3$ and $12.8 \pm 3.2$, respectively. Based on the APACHE-II scores, previous studies on in-hospital mortality rates reported mortality rates of $55 \%$ to $73 \%$ for more than 28 points, which were somewhat consistent

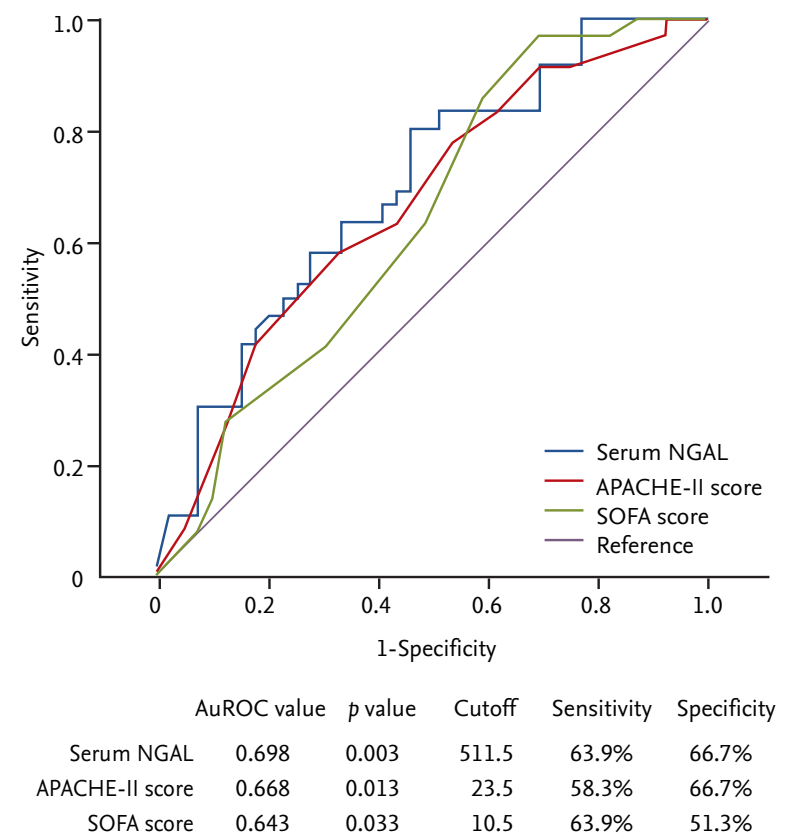

Figure 4. Predictive value for 28-day mortality of serum neutrophil gelatinase-associated lipocalin (NGAL) level, Acute Physiology and Chronic Health Evaluation-II (APACHE-II) score, and Sequential Organ Failure Assessment (SOFA) score in low APACHE-II score group. AuROC, area under the receiver operating characteristic curve.

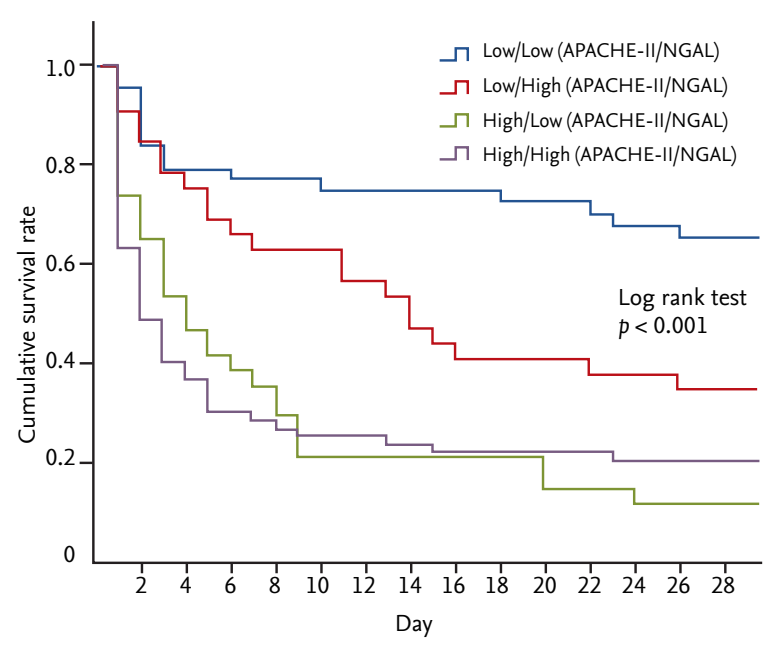

$\begin{array}{llllllllllllllll}\text { Low/Low } & 43 & 36 & 34 & 33 & 33 & 32 & 32 & 32 & 32 & 31 & 31 & 30 & 29 & 28 & 28\end{array}$ $\begin{array}{llllllllllllllll}\text { Low/High } & 32 & 27 & 24 & 21 & 21 & 21 & 18 & 15 & 13 & 13 & 13 & 12 & 12 & 11 & 11\end{array}$ $\begin{array}{llllllllllllllll}\text { High/Low } & 34 & 22 & 16 & 13 & 10 & 7 & 7 & 7 & 7 & 7 & 5 & 5 & 4 & 4 & 4\end{array}$ $\begin{array}{llllllllllllllll}\text { High/High } & 60 & 29 & 22 & 18 & 16 & 15 & 15 & 14 & 13 & 13 & 13 & 13 & 12 & 12 & 12\end{array}$

Figure 5. Kaplan-Meier survival analysis according to serum neutrophil gelatinase-associated lipocalin (NGAL) level and Acute Physiology and Chronic Health Evaluation-II (APACHE-II) score groups divided by cutoff value. 
Table 2. Multivariate Cox proportional hazard regression analysis of 28-day mortality in the low APACHE-II score group

\begin{tabular}{lccc}
\hline Variable & $\beta$ & Hazard ratio (95\% confidence interval) & $p$ value \\
\hline NGAL $\geq 511.5 \mathrm{ng} / \mathrm{mL}$ & 0.878 & $2.405(1.209-4.783)$ & 0.012 \\
APACHE-II score $\geq 23.5$ & 0.829 & $2.291(1.173-4.472)$ & 0.015 \\
\hline
\end{tabular}

Multivariate regression model was adjusted with parameters showing significant difference between the survivor and non-survivor groups. Parameters were as follows: NGAL group according to cutoff value (511.5 ng/mL), APACHE-II score group according to cutoff value (23.5), Sequential Organ Failure Assessment (SOFA) score group according to cutoff value (10.5), age, inotropic agent administration, and indication for continuous renal replacement therapy initiation (sepsis).

APACHE-II, Acute Physiology and Chronic Health Evaluation-II; NGAL, neutrophil gelatinase-associated lipocalin.

with our findings $[15,19,20]$.

Several studies have previously analyzed mortality prediction values of serum NGAL levels in critically ill patients with AKI. Kumpers et al. [21] analyzed the relationship between serum NGAL levels and 28-day mortality in 109 critically ill patients with AKI requiring renal replacement therapy; serum NGAL levels were reported to be an independent risk factor predicting 28 -day mortality (hazard ratio, 1.6; $p=0.005$ ). Mahmoodpoor et al. [13] analyzed the mortality prediction values of both serum and urinary NGAL levels in 50 patients with AKI. The AuROC value of serum NGAL at the time of admission to the intensive care unit was reported as 0.587 . In addition, the authors analyzed the mortality prediction value according to the change in serum NGAL levels after 48 hours, and the AuROC value was 0.874 . These studies were limited due to a relatively small sample size.

In this study, serum NGAL levels across all study groups resulted in lower prediction power than APACHE-II scores and SOFA scores, which are well known mortality prediction markers for critically ill patients and are calculated using factors directly related to patient mortality $[15,16,22]$. On the other hand, serum NGAL levels reflect organ injury caused by ischemia-reperfusion, so the direct mortality prediction value may be relatively low as demonstrated in this study [4]. In addition, since this study focused on patients with AKI, many patients had high baseline serum NGAL levels which may have influenced the results. Furthermore, APACHE-II scores, SOFA scores, and serum NGAL levels were cross-sectionally analyzed only at the time of CRRT initiation and it is possible that the tests were performed in some cases after serum NGAL levels had decreased following the occurrence of AKI [23]. These reasons could also explain the poor correlation between serum NGAL levels and the other two scoring systems as shown in our study.

However, our study also showed that in the lower APACHE-II score group, serum NGAL levels tended to show better predictive values for 28-day mortality compared with the APACHE-II and SOFA scoring systems. Furthermore, serum NGAL level was an independent risk factor for mortality in the multivariate Cox regression analysis. NGAL was first discovered in mature neutrophil granules [1] but is now known to be expressed in the proximal and distal tubular cells in the kidney [24], hepatocytes [25], smooth muscle cells [26], cardiomyocytes [27], endothelial cells [28], neurons [29], and various immune cells [5,30]. NGAL levels are thought to increase in these cells in the early stages of organ damage in ischemia-reperfusion injury. In addition, NGAL itself has been reported to stimulate the NF- $\mathrm{KB}$ pathway to increase the expression of various proinflammatory molecules [31]. Thus, increased concentration of NGAL itself may have increased inflammation which may have affected patient mortality. Anyway, elevated NGAL levels may be helpful in predicting mortality because they reflect the extent of subclinical organ damage in patients whose APACHE-II or SOFA scores have not yet increased.

Previous studies have shown that NGAL is difficult to use as an AKI predictor in septic conditions because it is expressed in several inflammatory cells. [2,4]. As such, the presence of sepsis in patients may affect serum NGAL levels. In this study, a large number of sepsis patients were included in the non-survivor group; therefore, subgroup analysis was performed. Considering the entire patient group, the AuROC value of serum NGAL was 0.658 with statistical significance only in the non-sepsis group. However, in the low APACHE-II score group, the AuROC value of serum NGAL was 0.620 and 0.650 of the non-sepsis and sepsis groups, respectively, which were 
lower than that of all low APACHE-II patient groups taken together (Supplementary Fig. 1). Notably, in the low APACHE-II score group, the non-survivor group contained more sepsis patients, and the serum NGAL level was found to be a significant factor affecting mortality even if the presence of sepsis was corrected by multivariate analysis (Table 2).

This study had some limitations. Firstly, this was a single-center, retrospective study with a small sample size of 169 patients. It is possible that this small number of patients did not sufficiently represent the population. However, our study has the advantage that the sample size is larger than in previous studies that reported the relationship between serum NGAL level and mortality in critically ill patients with AKI $[13,21]$. Secondly, serum NGAL levels were measured and analyzed only once (at the time of CRRT initiation). Previous studies have reported that subsequent measurements of serum NGAL levels may be of greater help in predicting mortality $[11,13]$. In this study, serial follow-up of serum NGAL levels was not performed due to various environmental limitations. However, this study suggests that serum NGAL levels at the time of CRRT initiation may be useful for predicting mortality in patients with low clinical severity.

In conclusion, in critically ill patients with AKI requiring CRRT, serum NGAL levels were shown to be a useful prediction marker for short term mortality in patients with relatively low clinical severity. These results indicate that subclinical tissue damage may be associated with high serum NGAL levels before the development of obvious signs of multiorgan failure. Baseline serum NGAL levels in patients requiring CRRT with relatively low clinical severity may be useful markers for predicting mortality in the future.

\section{KEY MESSAGE}

1. Neutrophil gelatinase-associated lipocalin (NGAL) secretion increases when various types of cells are injured.

2. Serum NGAL is increased by injury to various organs not only the kidney.

3. Serum NGAL is an early indicator of organ injury with a low clinical severity.

4. In relatively low clinical severity, serum NGAL is a useful predictor of mortality.

\section{Conflict of interest}

No potential conflict of interest relevant to this article was reported.

\section{REFERENCES}

1. Kjeldsen L, Johnsen AH, Sengelov H, Borregaard N. Isolation and primary structure of NGAL, a novel protein associated with human neutrophil gelatinase. J Biol Chem 1993;268:10425-10432.

2. Ronco C, Legrand M, Goldstein SL, et al. Neutrophil gelatinase-associated lipocalin: ready for routine clinical use? An international perspective. Blood Purif 2014;37:271285.

3. Lippi G, Meschi T, Nouvenne A, Mattiuzzi C, Borghi L. Neutrophil gelatinase-associated lipocalin in cancer. Adv Clin Chem 2014;64:179-219.

4. Paragas N, Qiu A, Zhang Q, et al. The Ngal reporter mouse detects the response of the kidney to injury in real time. Nat Med 2011;17:216-222.

5. Otto GP, Hurtado-Oliveros J, Chung HY, et al. Plasma neutrophil gelatinase-associated lipocalin is primarily related to inflammation during sepsis: a translational approach. PLoS One 2015;10:e0124429.

6. Cantaluppi V, Tamagnone M, Dellepiane S, Medica D. Plasma NGAL is an early biomarker of graft function, calcineurin inhibitor nephrotoxicity and tubular regeneration in kidney transplantation from extended criteria donors: 902. Transplantation 2012;94(10 Suppl):1168.

7. Cruz DN, Gaiao S, Maisel A, Ronco C, Devarajan P. Neutrophil gelatinase-associated lipocalin as a biomarker of cardiovascular disease: a systematic review. Clin Chem Lab Med 2012;50:1533-1545.

8. Alvelos M, Lourenco P, Dias C, et al. Prognostic value of neutrophil gelatinase-associated lipocalin in acute heart failure. Int J Cardiol 2013;165:51-55.

9. Hemdahl AL, Gabrielsen A, Zhu C, et al. Expression of neutrophil gelatinase-associated lipocalin in atherosclerosis and myocardial infarction. Arterioscler Thromb Vasc Biol 2006;26:136-142.

10. Lee CW, Kou HW, Chou HS, et al. A combination of SOFA score and biomarkers gives a better prediction of septic AKI and in-hospital mortality in critically ill surgical patients: a pilot study. World J Emerg Surg 2018;13:41.

11. Chang W, Zhu S, Pan C, et al. Predictive utilities of neu- 
trophil gelatinase-associated lipocalin (NGAL) in severe sepsis. Clin Chim Acta 2018;481:200-206.

12. Wang B, Chen G, Zhang J, Xue J, Cao Y, Wu Y. Increased neutrophil gelatinase-associated lipocalin is associated with mortality and multiple organ dysfunction syndrome in severe sepsis and septic shock. Shock 2015;44:234-238.

13. Mahmoodpoor A, Hamishehkar H, Fattahi V, Sanaie S, Arora P, Nader ND. Urinary versus plasma neutrophil gelatinase-associated lipocalin (NGAL) as a predictor of mortality for acute kidney injury in intensive care unit patients. J Clin Anesth 2018;44:12-17.

14. Kellum JA, Lameire N, Aspelin P, et al. Kidney disease: improving global outcomes (KDIGO) acute kidney injury work group. KDIGO clinical practice guideline for acute kidney injury. Kidney Int Suppl 2012;2:1-138.

15. Knaus WA, Draper EA, Wagner DP, Zimmerman JE. APACHE II: a severity of disease classification system. Crit Care Med 1985;13:818-829.

16. Vincent JL, Moreno R, Takala J, et al. The SOFA (Sepsis-related Organ Failure Assessment) score to describe organ dysfunction/failure. On behalf of the Working Group on Sepsis-Related Problems of the European Society of Intensive Care Medicine. Intensive Care Med 1996;22:707710.

17. Seo Y, Lee W, Kwon OH. Peformance evaluation of bioporto diagnostics' neutrophil gelatinase-associated lipocalin assay on automated clinical chemistry analyzer Hitachi 7600. J Lab Med Qual Assur 2013;35:23-28.

18. Krzanowski WJ, Hand DJ. ROC Curves for Continuous Data. Boca Raton (FL):CRC Press, 2009.

19. Chiavone PA, Sens YA. Evaluation of APACHE II system among intensive care patients at a teaching hospital. Sao Paulo Med J 2003;121:53-57.

20. Naved SA, Siddiqui S, Khan FH. APACHE-II score correlation with mortality and length of stay in an intensive care unit. J Coll Physicians Surg Pak 2011;21:4-8.

21. Kumpers P, Hafer C, Lukasz A, et al. Serum neutrophil gelatinase-associated lipocalin at inception of renal replacement therapy predicts survival in critically ill patients with acute kidney injury. Crit Care 2010;14:R9.

22. Marsh HM, Krishan I, Naessens JM, et al. Assessment of prediction of mortality by using the APACHE II scoring system in intensive-care units. Mayo Clin Proc 1990;65:1549-1557.

23. Mishra J, Dent C, Tarabishi R, et al. Neutrophil gelatinase-associated lipocalin (NGAL) as a biomarker for acute renal injury after cardiac surgery. Lancet 2005;365:12311238.

24. Tajima S, Yamamoto N, Masuda S. Clinical prospects of biomarkers for the early detection and/or prediction of organ injury associated with pharmacotherapy. Biochem Pharmacol 2019;170:113664.

25. Borkham-Kamphorst E, Drews F, Weiskirchen R. Induction of lipocalin-2 expression in acute and chronic experimental liver injury moderated by pro-inflammatory cytokines interleukin-1 $\beta$ through nuclear factor- $\mathrm{\kappa}$ activation. Liver Int 2011;31:656-665.

26. Eilenberg W, Stojkovic S, Piechota-Polanczyk A, et al. Neutrophil gelatinase-associated lipocalin (NGAL) is associated with symptomatic carotid atherosclerosis and drives pro-inflammatory state in vitro. Eur J Vasc Endovasc Surg 2016;51:623-631.

27. Latouche C, El Moghrabi S, Messaoudi S, et al. Neutrophil gelatinase-associated lipocalin is a novel mineralocorticoid target in the cardiovascular system. Hypertension 2012;59:966-972.

28. Hamzic N, Blomqvist A, Nilsberth C. Immune-induced expression of lipocalin-2 in brain endothelial cells: relationship with interleukin-6, cyclooxygenase-2 and the febrile response. J Neuroendocrinol 2013;25:271-280.

29. Naude PJ, Eisel UL, Comijs HC, et al. Neutrophil gelatinase-associated lipocalin: a novel inflammatory marker associated with late-life depression. J Psychosom Res 2013;75:444-450.

30. Flo TH, Smith KD, Sato S, et al. Lipocalin 2 mediates an innate immune response to bacterial infection by sequestrating iron. Nature 2004;432:917-921.

31. Bu DX, Hemdahl AL, Gabrielsen A, et al. Induction of neutrophil gelatinase-associated lipocalin in vascular injury via activation of nuclear factor-kappaB. Am J Pathol 2006;169:2245-2253. 
Supplementary Table 1. Baseline characteristics and clinical features of survivor and non-survivor group in the low APACHEII score group $(<27 \cdot 5)$

\begin{tabular}{|c|c|c|c|}
\hline Characteristic & Survivor $(\mathrm{n}=39)$ & Non-survivor $(\mathrm{n}=36)$ & $p$ value \\
\hline Serum NGAL, ng/mL & $383.0(215.0-713.0)$ & $716.0(397.5-1,239.8)$ & 0.003 \\
\hline APACHE-II score & $22.0(16.0-24.0)$ & $24.0(22.0-26.0)$ & 0.012 \\
\hline SOFA score & $10.0(8.0-12.0)$ & $11.0(10.0-13.0)$ & 0.032 \\
\hline Serum NGAL $\geq 511.5 \mathrm{ng} / \mathrm{mL}$ & $13(33 \cdot 3)$ & $23(63.9)$ & 0.008 \\
\hline APACHE-II score $\geq 23.5$ & $13(33 \cdot 3)$ & $21(58.3)$ & 0.030 \\
\hline SOFA score $\geq 10.5$ & $19(48.7)$ & $23(63.9)$ & 0.186 \\
\hline Age, yr & $57.1 \pm 17.1$ & $64.8 \pm 15 \cdot 9$ & 0.047 \\
\hline Body mass index, $\mathrm{kg} / \mathrm{m}^{2}$ & $23.6 \pm 3.9$ & $22.3 \pm 3.6$ & 0.168 \\
\hline Male sex & $24(61.5)$ & $19(52.8)$ & 0.443 \\
\hline C-reactive protein, mg/dL & $9.5(2.7-18.4)$ & $13.5(8.1-18.5)$ & 0.360 \\
\hline Mechanical ventilation & $15(38.5)$ & $20(55 \cdot 6)$ & 0.138 \\
\hline Inotropics administration & $18(46.2)$ & $25(69.4)$ & 0.042 \\
\hline \multicolumn{4}{|l|}{ Indication for CRRT initiation } \\
\hline Sepsis & $13(33 \cdot 3)$ & $22(61.1)$ & 0.016 \\
\hline Postoperation & $7(17 \cdot 9)$ & $1(2.8)$ & 0.058 \\
\hline Cardiac & $4(10.3)$ & $4(11.1)$ & 1.000 \\
\hline Pulmonary & $\mathrm{O}$ & $1(2.8)$ & 0.480 \\
\hline Hepatic & $8(20.5)$ & $3(8.3)$ & 0.136 \\
\hline Renal & $2(5 \cdot 1)$ & $2(5 \cdot 6)$ & 1.000 \\
\hline Malignancy & $5(12.8)$ & $3(8.3)$ & 0.713 \\
\hline Others & $\mathrm{O}$ & $\mathrm{O}$ & - \\
\hline \multicolumn{4}{|l|}{ Comorbidities } \\
\hline Diabetes mellitus & $14(35 \cdot 9)$ & $9(25 \cdot 0)$ & 0.307 \\
\hline Hypertension & $14(35 \cdot 9)$ & $18(50.0)$ & 0.217 \\
\hline Chronic kidney disease & $6(15 \cdot 4)$ & $6(16.7)$ & 0.880 \\
\hline Cerebro-cardiovascular disease & $12(30.8)$ & $10(27.8)$ & 0.776 \\
\hline Pulmonary disease & $1(2.6)$ & $1(2.8)$ & 1.000 \\
\hline Liver disease & $12(30.8)$ & $5(13.9)$ & 0.081 \\
\hline Dementia & o & $1(2.8)$ & 0.480 \\
\hline Malignancy & $7(17 \cdot 9)$ & $12(33 \cdot 3)$ & 0.126 \\
\hline
\end{tabular}

Values are presented as median (interquartile range), number (\%), or mean $\pm \mathrm{SD}$.

APACHE-II, Acute Physiology and Chronic Health Evaluation-II; NGAL, neutrophil gelatinase-associated lipocalin; SOFA, Sequential Organ Failure Assessment; CRRT, continuous renal replacement therapy. 
Supplementary Table 2. Baseline characteristics and clinical features of survivor and non-survivor group in the high APACHE-II score group $(\geq 27 \cdot 5)$

\begin{tabular}{|c|c|c|c|}
\hline Characteristic & Survivor $(n=16)$ & Non-survivor $(\mathrm{n}=78)$ & $p$ value \\
\hline Serum NGAL, ng/mL & $797.5(548.8-1,370.5)$ & $759 \cdot 5(394.5-1,446.0)$ & 0.864 \\
\hline APACHE-II score & $30.0(28.0-35.0)$ & $33.0(30.8-37.0)$ & 0.040 \\
\hline SOFA score & $14.0(12.3-15.8)$ & $15.0(13.0-17.0)$ & 0.137 \\
\hline Serum NGAL $\geq 805.0 \mathrm{ng} / \mathrm{mL}$ & $7(43.8)$ & $38(48.7)$ & 0.717 \\
\hline APACHE-II score $\geq 32.5$ & $5(31.3)$ & $45(57 \cdot 7)$ & 0.053 \\
\hline SOFA score $\geq 14.5$ & $7(43.8)$ & $45(57 \cdot 7)$ & 0.307 \\
\hline Age, yr & $64.7 \pm 12.4$ & $65 \cdot 5 \pm 14.9$ & 0.839 \\
\hline Body mass index, $\mathrm{kg} / \mathrm{m}^{2}$ & $24.1(20.9-27.9)$ & $22.7(20.7-24.9)$ & 0.337 \\
\hline Male sex & $7(43.8)$ & $46(59.0)$ & 0.263 \\
\hline C-reactive protein, mg/dL & $13 \cdot 3(5.1-20.8)$ & $11.1(3.7-19.9)$ & 0.782 \\
\hline Mechanical ventilation & $10(62.5)$ & $70(89.7)$ & 0.013 \\
\hline Inotropics administration & $14(87.5)$ & $68(87.2)$ & 1.000 \\
\hline \multicolumn{4}{|l|}{ Indication for CRRT initiation } \\
\hline Sepsis & $9(56.3)$ & $49(62.8)$ & 0.622 \\
\hline Postoperation & $1(6.3)$ & $5(6.4)$ & 1.000 \\
\hline Cardiac & $1(6.3)$ & $8(10.3)$ & 1.000 \\
\hline Pulmonary & $1(6.3)$ & $1(1.3)$ & 0.313 \\
\hline Hepatic & $2(12.5)$ & $7(9.0)$ & 0.647 \\
\hline Renal & $2(12.5)$ & $3(3.8)$ & 0.200 \\
\hline Malignancy & 0 & $3(3.8)$ & 1.000 \\
\hline Others & o & $2(2.6)$ & 1.000 \\
\hline \multicolumn{4}{|l|}{ Comorbidities } \\
\hline Diabetes mellitus & $4(25 \cdot 0)$ & $19(24.4)$ & 1.000 \\
\hline Hypertension & $7(43.8)$ & $35(44 \cdot 9)$ & 0.934 \\
\hline Chronic kidney disease & $3(18.8)$ & $6(7 \cdot 7)$ & 0.178 \\
\hline Cerebro-cardiovascular disease & $5(31.3)$ & $18(23.1)$ & 0.529 \\
\hline Pulmonary disease & $1(6.3)$ & $8(10.3)$ & 1.000 \\
\hline Liver disease & $4(25 \cdot 0)$ & $13(16.7)$ & 0.479 \\
\hline Dementia & o & $1(1.3)$ & 1.000 \\
\hline Malignancy & $6(37.5)$ & $17(21.8)$ & 0.208 \\
\hline
\end{tabular}

Values are presented as median (interquartile range), number (\%), or mean $\pm \mathrm{SD}$.

APACHE-II, Acute Physiology and Chronic Health Evaluation-II; NGAL, neutrophil gelatinase-associated lipocalin; SOFA, Sequential Organ Failure Assessment; CRRT, continuous renal replacement therapy. 
Park Y, et al. Mortality predictive value of serum NGAL

Supplementary Table 3. Comparison of baseline characteristics between enrolled and excluded patient group

\begin{tabular}{|c|c|c|c|}
\hline Characteristic & Enrolled group $(\mathrm{n}=169)$ & Excluded group $(\mathrm{n}=335)$ & $p$ value \\
\hline 28-Day mortality & $114(67.5)$ & $220(65.7)$ & 0.689 \\
\hline Age, yr & $63.3 \pm 15 \cdot 7$ & $62.1 \pm 16.5$ & 0.426 \\
\hline Male sex & $73(43.2)$ & $127(37.9)$ & 0.252 \\
\hline Arterial pH & $7 \cdot 31 \pm 0.14$ & $7.30 \pm 0.22$ & 0.879 \\
\hline Serum sodium, mEq/L & $140.3 \pm 8.7$ & $139.9 \pm 8.3$ & 0.687 \\
\hline Serum potassium, $\mathrm{mEq} / \mathrm{L}$ & $4.3 \pm 0.9$ & $4.4 \pm 0.9$ & 0.543 \\
\hline Urea nitrogen, mg/dL & $61.5 \pm 31.2$ & $63.3 \pm 35.6$ & 0.572 \\
\hline Serum creatinine, $\mathrm{mg} / \mathrm{dL}$ & $2.87 \pm 1.54$ & $2.99 \pm 2.24$ & 0.544 \\
\hline Hematocrit, \% & $27.7 \pm 5.8$ & $27.8 \pm 5.5$ & 0.763 \\
\hline WBC count, $\times 10^{3} / \mu \mathrm{L}$ & $19.5 \pm 83.1$ & $12.1 \pm 10.4$ & 0.251 \\
\hline Platelet count, $\times 10^{3} / \mu \mathrm{L}$ & $89.9 \pm 80.2$ & $98.4 \pm 85.8$ & 0.282 \\
\hline Total protein, mg/dL & $5.03 \pm 1.1$ & $5.01 \pm 1.0$ & 0.863 \\
\hline Albumin, mg/dL & $2.7 \pm 0.6$ & $2.7 \pm 0.5$ & 0.678 \\
\hline Aspartate aminotransferase, IU/L & $710.1 \pm 1,309.4$ & $693.8 \pm 1,335.4$ & 0.897 \\
\hline Alanine aminotransferase, IU/L & $376.0 \pm 903.4$ & $399.9 \pm 897.5$ & 0.780 \\
\hline Total bilirubin, mg/dL & $4.63 \pm 7.44$ & $4.59 \pm 7.38$ & 0.955 \\
\hline C-reactive protein, mg/dL & $13.16 \pm 10.17$ & $12.08 \pm 10.80$ & 0.321 \\
\hline \multicolumn{4}{|l|}{ Indication for CRRT initiation } \\
\hline Sepsis & $93(55 \cdot 0)$ & $172(51.3)$ & 0.434 \\
\hline Postoperation & $14(8.3)$ & $40(11.9)$ & 0.210 \\
\hline Cardiac & $17(10.1)$ & $47(14.0)$ & 0.206 \\
\hline Pulmonary & o & $2(0.6)$ & 0.553 \\
\hline Hepatic & $9(5 \cdot 3)$ & $11(3 \cdot 3)$ & 0.268 \\
\hline Renal & $20(11.8)$ & $28(8.4)$ & 0.209 \\
\hline Malignancy & $11(6.5)$ & $14(4 \cdot 2)$ & 0.255 \\
\hline Others & $2(1.2)$ & $12(3.6)$ & 0.156 \\
\hline
\end{tabular}

Values are presented as number (\%) or mean \pm standard deviation.

WBC, white blood cell; CRRT, continuous renal replacement therapy. 

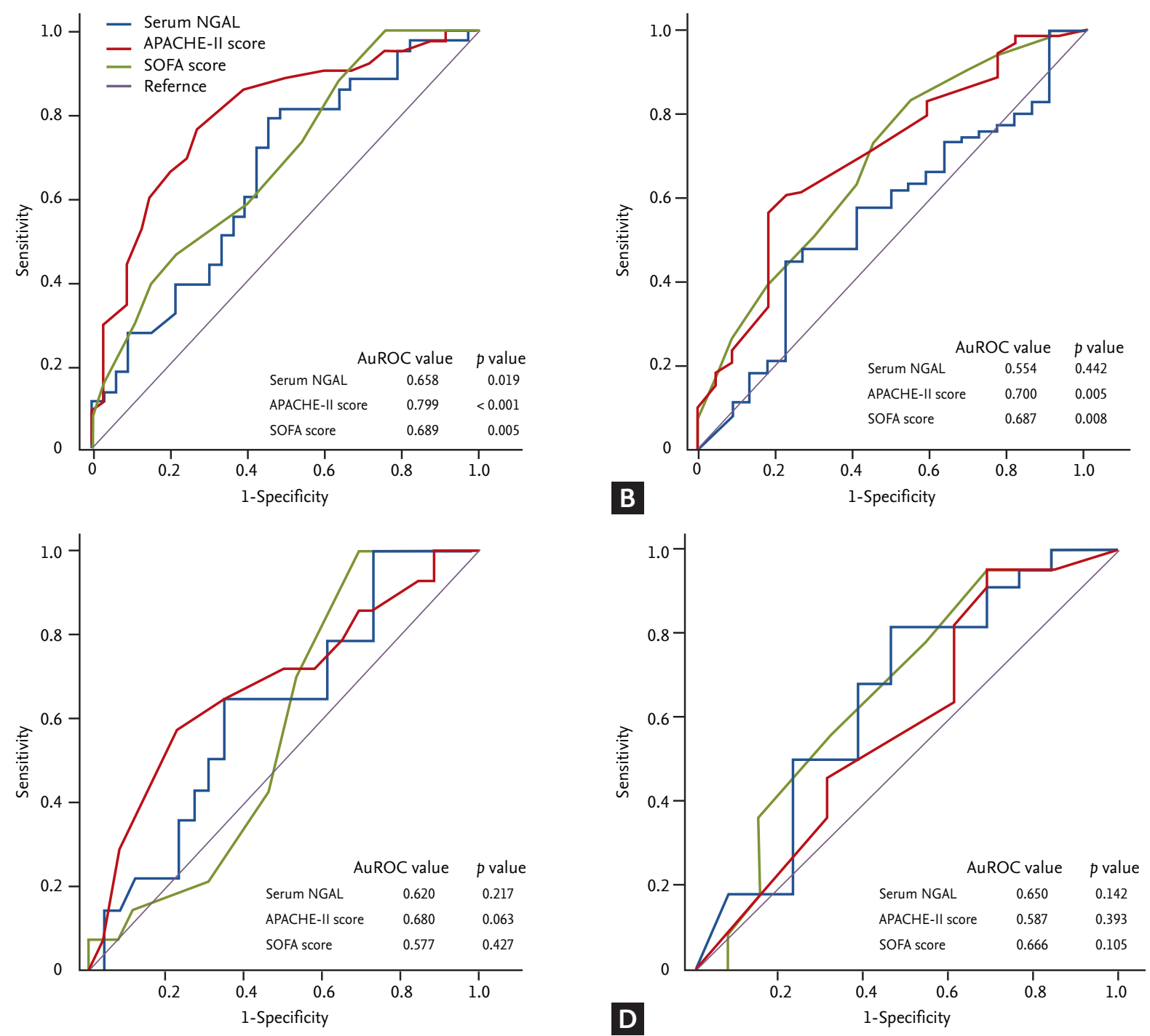

Supplementary Figure 1. Predictive value for 28-day mortality of serum neutrophil gelatinase-associated lipocalin (NGAL) level, Acute Physiology and Chronic Health Evaluation-II (APACHE-II) score, and Sequential Organ Failure Assessment (SOFA) score in subgroup analysis according to sepsis status. (A) Non-sepsis group in overall patients, (B) sepsis group in overall patients, (C) non-sepsis group in low APACHE-II score, and (D) sepsis group in low APACHE-II score. 